\title{
Feasibility of Speedboat RS2 with bipolar radiofrequency energy for peroral endoscopic myotomy in patients with achalasia (with video)
}

\section{(ㄷ)(i) $(=$}

\author{
Authors \\ Gaurav Patil, Anki Dalal, Amit P. Maydeo \\ Institution \\ Baldota Institute of Digestive Sciences, Gleneagles \\ Global Hospitals, Mumbai, Maharashtra, India \\ submitted 28.1.2020 \\ accepted after revision 1.4 .2020 \\ Bibliography \\ DOI https://doi.org/10.1055/a-1165-0087 | \\ Endoscopy International Open 2020; 08: E998-E1001 \\ (c) Georg Thieme Verlag KG Stuttgart · New York \\ eISSN 2196-9736 \\ Corresponding author \\ Prof. Amit Maydeo, Chairman, Baldota Institute of \\ Digestive Sciences, Gleneagles Global Hospitals, \\ Parel, Mumbai, Maharashtra 400012, India \\ Phone: +91 2267670311 \\ amitmaydeo@gmail.com
}

\section{Introduction}

Available devices and accessories for performing Per oral endoscopic myotomy (POEM) are sometimes technically challenging and require constant exchange for dissection and spot coagulation, resulting in prolonged procedural time with an acceptable risk of complications [1, 2]. Speedboat-RS2 (Creo-Medical, UK) is an endoscopic device that has been utilized for submucosal dissection of esophageal, gastric, and colonic lesions [3]. The device delivers bipolar radiofrequency energy (BRF) for dissection and cutting and microwave energy through its tip [4]. The integrated retractable injection needle is used for mucosal incision and submucosal lift, hence no instrument change is required. The CROMA generator is used to supply microwaves to ab-

\section{ABSTRACT}

Background During peroral endoscopic myotomy (POEM), use of traditional instruments leads to an increase in overall time of procedure due to constant exchange of instruments. Speedboat-RS2 (Creo-Medical, UK) is a novel device which has shown promising results for endoscopic submucosal dissection of gastrointestinal lesions, but its feasibility, and safety for POEM is unexplored.

Methods Data from patients who had undergone POEM for achalasia cardia utilizing Speedboat-RS2 was reviewed.

Results Two patients with mean age of 46 years and mean symptom duration of 3 years were included. Both patients had type II achalasia diagnosed on esophageal manometry. One patient underwent anterior myotomy and the other posterior approach. Submucosal tunneling $(\sim 15 \mathrm{~cm})$ was achieved in a mean of 20 minutes, myotomy $(\sim 9 \mathrm{~cm})$ in 8 minutes and the entire procedure was completed in a mean of 30 minutes with $100 \%$ technical success. Both patients were discharged the day after the procedure without any immediate adverse events. At 6 weeks, endoscopy was uneventful and the patients were asymptomatic.

Conclusions Early experience shows that Speedboat-RS2 is feasible for performing POEM with good safety profile. Submucosal tunneling was relatively quick and coagulation was effective. Long term studies with a larger patient cohort are warranted. late and coagulate. BRF is used for precise lateral and forward cutting [4]. Microwave coagulation helps to achieve controlled hemostasis, the heat-insulated hull reduces risk of muscle damage, and the optimized shaft design helps in controlled rotation. The combination of these features results in quicker procedures with good precision. The feasibility of this device for POEM is currently unexplored. We review our experience with initial two cases of POEM by using Speedboat-RS2 in patients with achalasia. 


\section{Methods}

This was a retrospective review of two patients who underwent POEM by using Speedboat-RS2 at a tertiary care referral center. The study conformed to the ethical guidelines of the 1975 Declaration of Helsinki (7th revision, 2013). The conduct of this study was approved by the Institutional Ethics Committee. Waiver of consent was obtained for the study. Both patients were adults and had previously given their written informed consent for the procedure.

The demographic details, procedural indications, clinical and physical findings of patients were extracted from their case records. Patients were kept nil per os for 12 hours prior to endoscopy. Proton pump inhibitors (PPIs), antiemetics, broadspectrum antibiotics, and premorbid medications (wherever appropriate) were administered an hour prior to the intervention. Two operators performed one procedure each. General anesthesia was used in both patients and the procedure was performed [5] with the patients in supine position.

We used a high-definition endoscope Evis Exera II GIF2TH180 gastrovideoscope (Olympus, United States). A retractable integrated needle was used for submucosal injection. After instillation of a mixture of $0.9 \%$ normal saline with $0.3 \%$ indigo carmine, a mucosal incision was made with the edge of the device using BRF. This was made along the 2 o'clock for anterior in the first case and 5 o'clock for posterior wall of the esophagus in the second case, $15 \mathrm{~cm}$ above the gastroesophageal junction (GEJ). The submucosal plane was entered and a submucosal tunnel was created (BRF, cutting frequency $400 \mathrm{kHz}$, power setting $35 \mathrm{~W}$ ) by using Speedboat-RS2 for $2 \mathrm{~cm}$ beyond the GEJ. The endoscope was withdrawn into the esophageal lumen and entered the stomach, where a bluish submucosal hue in a retroflexed view confirmed the adequacy of the tunnel. This step was repeated (if necessary) while dissecting beyond the GEJ. The Sppedboat-RS2's novel design provided lateral and/or forward cutting using the curved tip, flat edges of the blade. Microwave coagulation (frequency $5.8 \mathrm{GHz}$, power setting $10 \mathrm{~W}$ ) was used to achieve controlled hemostasis. Larger vessels were pre-coagulated with microwave power for 5 to $10 \mathrm{sec}$ onds. An insulated "hull" remained parallel to the muscle layer and there was no mucosal injury. The insulated hull is rotatable and can be oriented upside down. About $9 \mathrm{~cm}$ of the circular and longitudinal muscle fibers of the esophagus were cut effortlessly and a complete myotomy was performed $2 \mathrm{~cm}$ beyond the GEJ. The esophageal lumen was inspected for any mucosal break. The mucosal incision was finally closed with endoscopic clips (Instinct endoscopic hemoclip, Cook Medical, Bloomington, Indiana, United States ( $>$ Fig. $\mathbf{1 a - i}$ and $\triangleright$ Video 1). Patients were kept NPO for 24 hours and then discharged.

\section{Results}

Two patients (both males) with a mean age of 46 years and mean symptom duration of 3 years were included. Both patients had type II achalasia cardia diagnosed on high-resolution manometry (HRM) (Trace 1.2.3a V software, Royal Melbourne Hospital, Australia). One patient underwent anterior myotomy and the other posterior approach. Submucosal tunneling ( 15 $\mathrm{cm}$ ) was achieved in a mean of 20 minutes, and $\sim 9 \mathrm{~cm}$ myotomy in 8 minutes and the entire procedure was completed in a mean of 30 minutes with $100 \%$ technical success ( $\triangleright$ Table $\mathbf{1}$ ). Both patients were discharged the day after the procedure without any immediate complications. Both patients were tolerating soft diet, so they were advised to transition to solid food. At 6-week follow-up, both patients were asymptomatic, able to swallow solid food without difficulty, and underwent upper gastroscopy, which was uneventful.

\section{Discussion}

We used the Speedboat-RS2 to perform POEM in two patients and a good response was seen. We used different a myotomy approach in each patient. That was done to compare differences in length of time for the procedures. Both procedures were safely done in an endoscopy suite and the patients did not have any complications. We found that the device helps to reduce overall procedure time as we did not change the device for bleeding vessels which normally require a coagulation grasper.

Prior to the advent of the Speedboat-RS2, POEM could be performed with standard techniques in about 40 minutes. The main advantages of the device are reduced risk of injury to mucosa by orientation of the hull and use of BRF, which aids quick tissue healing, thereby resulting in quicker post-procedural recovery and time savings. The true benefit of using this device is that it makes life much easier during submucosal tunneling which is a crucial step in POEM. With its sophisticated design, the Speedboard-RS2 cuts in forward, lateral, and oblique planes using bipolar RF $400 \mathrm{KHz}$ cutting and provides hemostasis with microwave coagulation $(5.8 \mathrm{GHz})$. To the best of our knowledge, this is the first case report of use of the SpeedboatRS2 for POEM in the world and the first use of such a device in the Asia-Pacific region for third space endoscopy. The device has already met with success in en-bloc gastric/esophageal mucosal resection $[6,7]$. Although the economics of it currently remain unexplored, the device certainly looks to make a huge impact in terms of its advantages over currently existing standard accessories in POEM. Our center has been performing POEM for almost a decade and diverticular POEM [8], and the advent of Speedboat-RS2 has certainly opened up new avenues in third space endoscopy [9].

Although POEM has been practiced for more than a decade, major bleeding, pneumothorax, mucosal perforation, and post procedural infections still are routinely encountered. Although in the majority of cases, these complications can be tackled in the same sitting, some patients need repeated interventions so as to prevent future complications. This leads to patient dissatisfaction with the procedure. We feel the advanced technology of the Speedboat RS2 overcomes many of the problems faced in routine third space procedures. Bipolar devices are specialized, with a range of outputs and modes for various applications, especially in elderly patients with cardiac conditions [10]. Speedboat-RS2 has recently received approval from the US Food and Drug Administration for use in minimally invasive removal of bowel lesions. The device may be of vital use in var- 

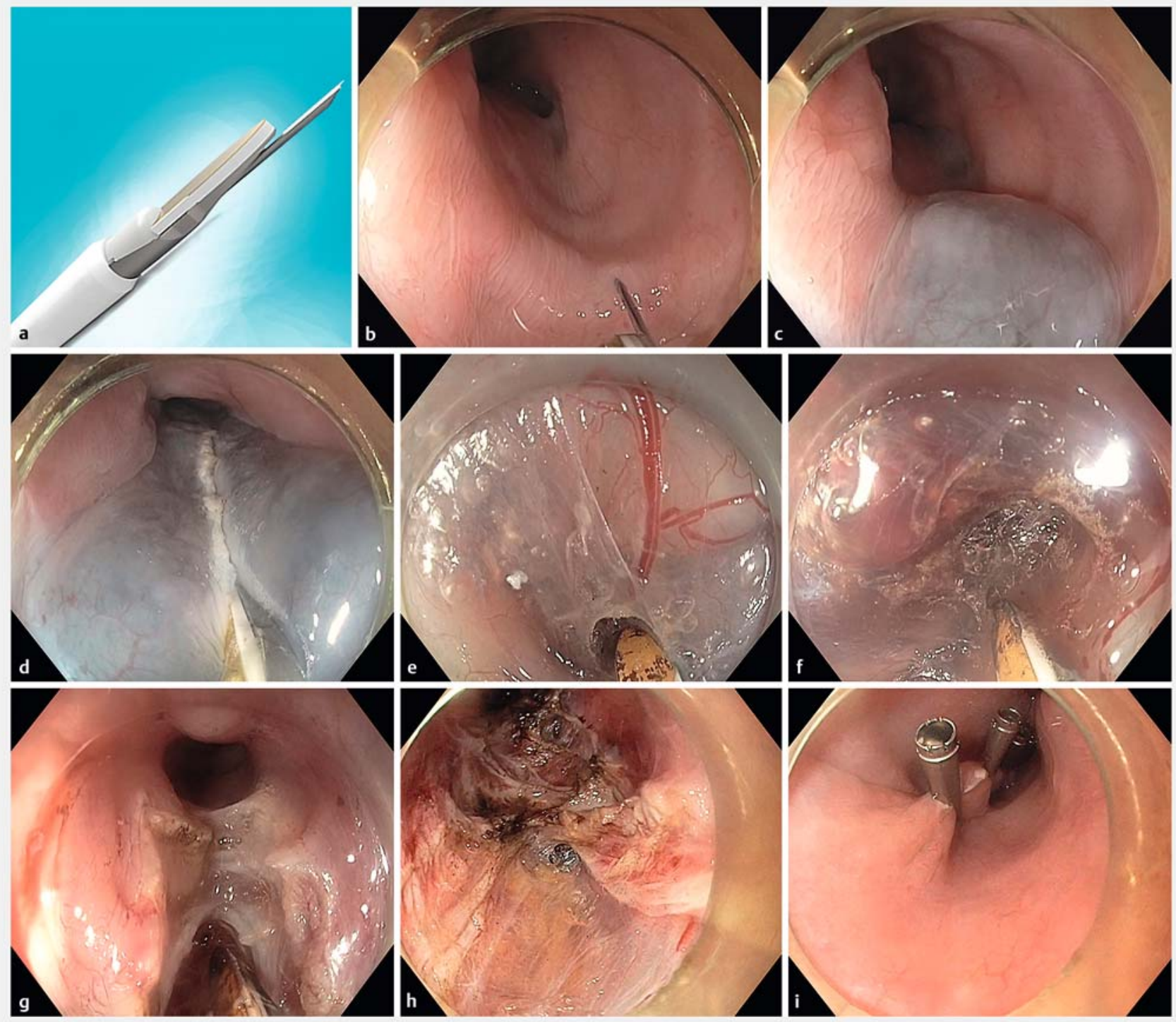

- Fig. 1 a Speedboat RS2 Endoscopic device with attached blade, insulated hull, and needle. b Retractable integrated needle being used for submucosal injection. $\mathbf{c}$ Instillation of saline underneath mucosa. $\mathbf{d}$ An incision being made on posterior wall of the esophagus. e Submucosal tunnel being created using Speedboat-RS2. $\mathbf{f}$ Lateral and forward cutting of circular and longitudinal muscle fibers of the esophagus using the curved tip and flat edges of the blade. $\mathbf{g}$ Lateral and forward cutting of circular and longitudinal muscle fibers of the esophagus using the curved tip and flat edges of the blade. $\mathbf{h}$ Lateral and forward cutting of circular and longitudinal muscle fibers of the esophagus using the curved tip and flat edges of the blade. $\mathbf{i}$ Mucosal incision closed with standard hemoclips.

ious minimally invasive surgical procedures [11]. While performing the procedure, we used a $2 \mathrm{TH}$ endoscope, since the Speedboat-RS2 needs a wider therapeutic channel $(3.7 \mathrm{~mm})$, however, the procedure can even be performed with a $1 \mathrm{TH}$ endoscope from Olympus. The latter was currently unavailable to us, hence we proceeded with the former. We did not encounter any flexibility issues when using a larger scope and POEM was performed in both patients meticulously and with ease.

Cutting of tissue and coagulating small vessels traditionally has been achieved by using monopolar current (high voltage $2000 \mathrm{~V}$ ) which has potential for resulting in deep burns causing mucosal perforation. The Speedboat-RS2, in contrast, uses bi- polar RF (low voltage 200V) for large vessels, which is associated with minimal risk of deep burns and helps with rapid tissue healing $[6,10]$. The device is boat-shaped and has curved bipolar electrodes on the sides. When current flows through the tissue between the two electrodes, the cutting effect is restricted to the needle electrode. When an incision is made, tissue damage is restricted to this small region. The power requirements are lower because the current is limited to the small piece of tissue between the active electrodes, which results in more predictable tissue modification [10]. Although utmost care is required when using the Speedboat-RS2 in a confined space, potential for alternative site burns is minimal. BRF indeed provides 


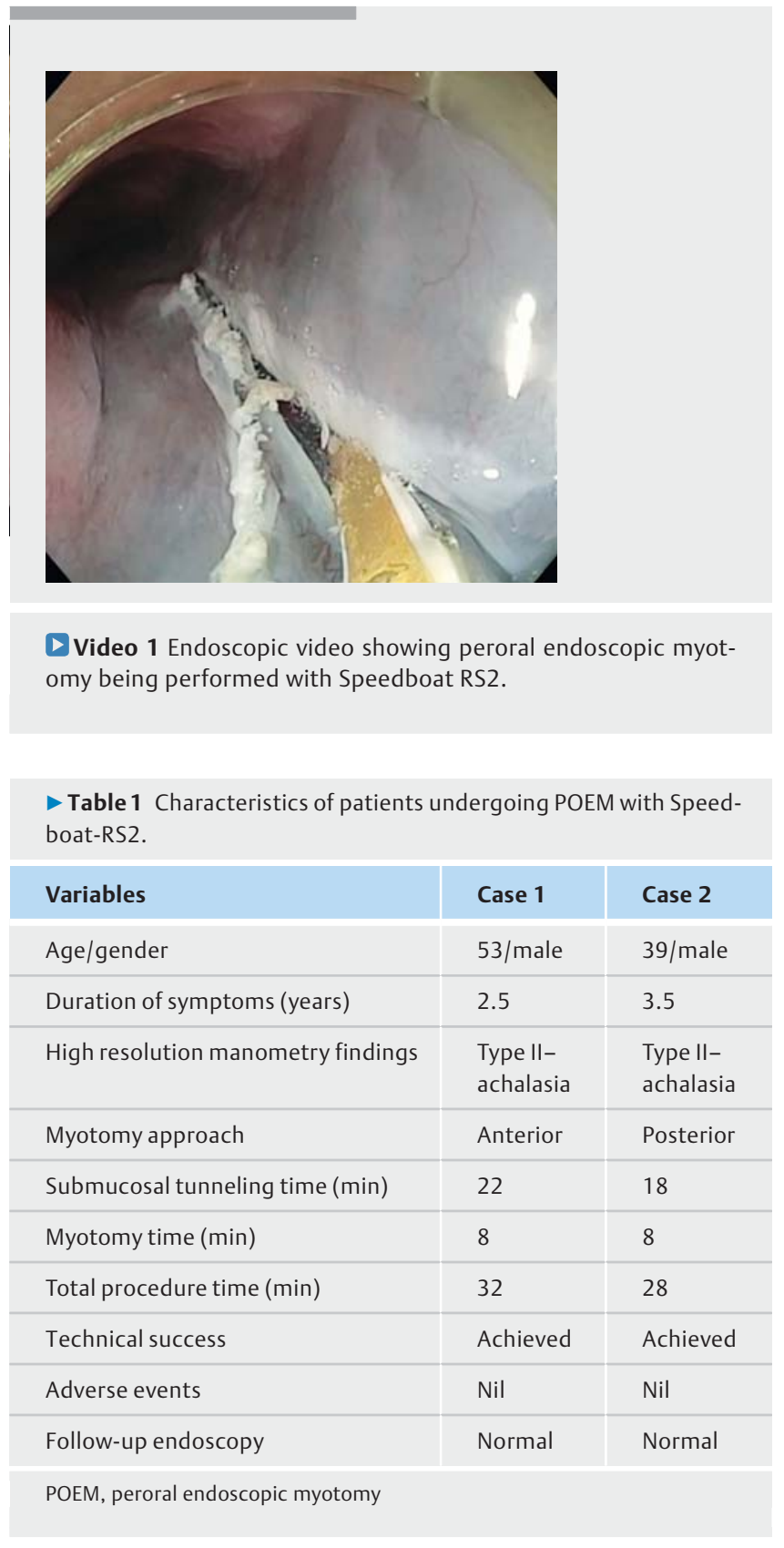

multiple advantages compared to stand-alone injection needles and cutting devices, but there exists an unmet need for future comparative studies with the hybrid knife device, which also can be used to inject and dissect. The latter uses a water jet for rapid submucosal infusion of saline solution while tunneling, eliminating the need for repeated changes of equipment. However, in the event of bleeding from larger vessels, especially at GEJ, coagrasper hemostatic forceps are required to achieve hemostasis. With the Speedboat device, hemostasis can be achieved with the same device because uses microwave coagulation and controlled hemostasis. It also limits the depth of injury. Doctors undergoing training or fellowship will find it much easier to use Speedboat RS2 for third space endoscopy as the device may reduce the overall risk and potential complications associated with these procedures. The small sample size is a limitation of our study, however, looking at the promising results with the device, we believe such anecdotal innovative cases lay a platform for developing future studies.

\section{Conclusion}

To conclude, early experience shows that use of the SpeedboatRS2 to perform POEM is feasible and it has a good safety profile and precision. Submucosal tunneling with the device was done relatively quickly and coagulation was effective. Long-term studies with a larger patient cohort are warranted.

\section{Competing interests}

The authors declare that they have no conflict of interest.

\section{References}

[1] Tang X, Ren Y, Wei Z et al. Factors predicting the technical difficulty of peroral endoscopic myotomy for achalasia. Surg Endosc 2016; 30 : 3774-3782

[2] Tan Y, Li C, Yan J et al. Difficult peroral endoscopic myotomy: definition and management strategies. Expert Rev Gastroenterol Hepatol 2019; 13: 933-942

[3] Saunders BP, Tsiamoulos ZP, Bourikas L et al. The "Speedboat": a new multi-modality instrument for endoscopic resection in the gastrointestinal tract. Gastrointest Endosc 2013; 77: AB155

[4] Tsiamoulos ZP, Sebastian J, Bagla N et al. A new approach to endoscopic submucosal tunneling dissection: the "Speedboat-RS2" device. Endoscopy 2019; 51: E185-E186

[5] Maydeo AP, Joshi N], Bhandari SP. Per oral endoscopic myotomy for a patient with achalasia cardia. J Assoc Physicians India 2012; 60: 66-68

[6] Tsiamoulos ZP, Stasinos I, Oikonomakis A et al. ATH-06. First clinical experience with speedboat-RS2, a new multimodality device for colonic submucosal dissection. Gut 2019; 68: A7-A8

[7] Tsiamoulos ZP, Hancock CP, Bourikas L et al. Sa1567. The "SpeedboatRS2": a new multi-modality endoscopic device for gastric and oesophageal submucosal dissection and tunneling. Gastrointest Endosc 2014; 79: AB257-AB258

[8] Maydeo A, Patil GK, Dalal A. Operative technical tricks and 12-month outcomes of diverticular peroral endoscopic myotomy (D-POEM) in patients with symptomatic esophageal diverticula. Endoscopy 2019; 51: $1136-1140$

[9] Inoue H, Maydeo A. Peroral endoscopic myotomy (POEM) opens the door of third-space endoscopy. Endoscopy 2019; 51: 1010-1012

[10] Bipolar Electrosurgery Review. MDA evaluation 01022, March 2001 Available at (Accessed: 10 January 2020): http://www.cedar.wales. nhs.uk/sitesplus/documents/1091/01022\%20Bipolar\%20electrosurgery\%20review\%202001.pdf

[11] Creo Medical's Speedboat RS2. Available at (Accessed: 10 January 2020): https://investors.creomedical.com/what-we-do/our-products 